\title{
An investigation of errors in estimates of the cometary nuclei active area fractions
}

\author{
P. J. Gutiérrez ${ }^{1}$, R. Rodrigo ${ }^{1}$, J. L. Ortiz ${ }^{1}$, and B. J. R. Davidsson ${ }^{2}$ \\ 1 Instituto de Astrofísica de Andalucía, CSIC, Aptd 3004, 18080 Granada, Spain \\ ${ }^{2}$ Department of Astronomy and Space Physics, Box 515, 75120 Uppsala, Sweden
}

Received 26 September 2002 / Accepted 28 January 2003

\begin{abstract}
Active area fractions of cometary nuclei are often estimated by comparing the observed water production rates with theoretical rates obtained by applying the fast rotator or subsolar point approximations to spherical model nuclei. Any discrepancy between observed and theoretical production rates is interpreted as a certain degree of dust mantling (or in some cases hyper activity) of the object. We here investigate the typical errors introduced in such active area fraction estimates by the usage of oversimplified spherical model nuclei. This is done by first calculating the production rates of slowly rotating irregular model bodies with different activity patterns on their surfaces and arbitrary spin axis orientations, for which solar illumination is treated properly. Next, the production rates of the spherical model objects under averaged insolation are compared to the production rates of the complex model objects in an attempt to recover the known active area fraction of the latter bodies. We then find that the fast rotator and subsolar point approximations generally yield large over- and underestimates of the active area fraction, depending on the characteristics of the simulated complex nuclei. Acceptable relative errors $(<100 \%)$ only occur at small heliocentric distances, and the subsolar point approximation yields somewhat better results than the fast rotator approximation.
\end{abstract}

Key words. comets: general - solar system: general

\section{Introduction}

Many of the cometary models developed so far (e.g. Fanale \& Salvail 1984, 1987; Enzian et al. 1997; Davidsson \& Skorov 2002 , etc.) consider the nucleus as a slowly rotating spherical body, where local and time-dependent illumination conditions are fully accounted for. On the other hand, several models use rotational-averaged illumination, either for individual latitudinal slabs (e.g., Orosei et al. 1995; Capria et al. 1996; De Sanctis et al. 1999) or for the whole nucleus, such as in the fast rotator and subsolar point approximations (e.g., Rickman et al. 1990; Prialnik et al. 1993; Tancredi et al. 1994; Benkhoff \& Huebner 1995).

In the fast rotator approximation, the nucleus is assumed to be a spherical body for which the energy flux is uniformly distributed over the entire surface. In the subsolar point approximation, it is assumed that the water production rate of the nucleus is given by the production rate at the subsolar point multiplied by the mean cross section of the nucleus.

Regardless of the complexity of a thermophysical model, the accuracy (in terms of water production rates) of the models is determined by their capability to correctly distribute the available solar energy between the main energy sinks - sublimation, thermal reradiation and conductivity. For low insolation fluxes, the latter two terms gradually gain importance,

Send offprint requests to: P. J. Gutiérrez, e-mail: pedroj@iaa.es since sublimation wears off rapidly with decreasing surface temperature. The fast rotator approximation is therefore expected to put a too large weight on thermal reradiation and conductivity compared to sublimation, since the available solar energy is smeared out over the whole surface of the sphere. The subsolar point approximation, on the other hand, overestimates the fraction of the solar energy consumed by sublimation, by neglecting the presence of shallowly illuminated (cold) areas on the sphere.

It can be shown that both approximations give very different water production rates from the water production obtained when a proper treatment of illumination geometry is used. In addition, most cometary nuclei are highly irregular and may have patchy activity patterns, which increases the complexity further. Two irregular bodies with the same illuminated cross sections (thus absorbing the same amount of solar energy) may have substantially different gas production rates, due to the differing surface topography. Locally, the relative importance of sublimation, reradiation, and conductivity as energy sinks depend on the surface inclination with respect to the Sun in a strongly non-linear manner. The global production rate can therefore not be obtained accurately by considering averaged quantities - an integration over properly calculated local rates is required.

As irregular bodies rotate, the gas production rate may vary considerably with time due to changes in the illuminated 

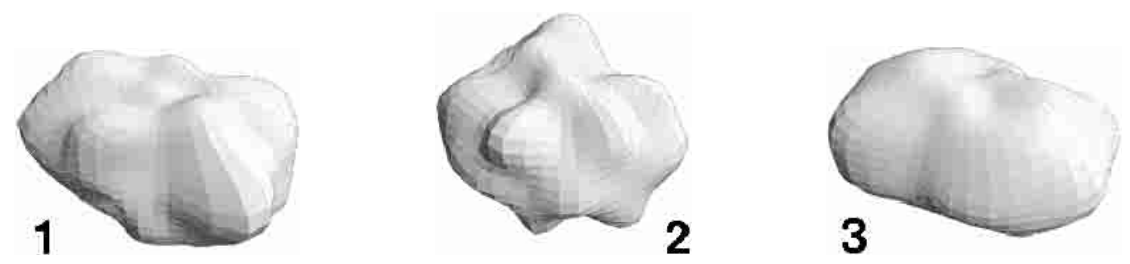

Fig. 1. Three Gaussian random shapes used in the simulations. For computational requirements, the surface of each objects has been divided in 1520 triangular cells.

cross section and the corresponding topography. The rotationally averaged gas production rate of an irregular body can therefore be quite different from that of a sphere, even if the bodies have the same volume, surface area, or mean cross section. Nevertheless, both approximate spherical models are commonly used in order to interpret observations in terms of properties of real cometary nuclei. For example, both approximations have been used to estimate the active nucleus area or, when the nucleus size is known with some certainty, the active area fraction (e.g., de Almeida et al. 1997; Rickman \& Jorda 1998; Fink et al. 1998; Möhlmann 1999; Benkhoff 1999; Sanzovo et al. 2001). We note that several investigations of this kind involve target comets for space missions such as Rosetta and Stardust, and that the results regarding nuclear activity may affect the planning of such missions.

To our knowledge, no previous study has been made in order to evaluate the errors in estimates of the cometary nuclei active area fractions. As a first step in evaluating errors associated with both approximations, we focus on the typical errors introduced by the simplified treatment of illumination conditions and the non consideration of complex nucleus shapes. In this paper, we compare the water production rates of slowly rotating irregular bodies with different activity patterns on their surfaces, with the water production rates calculated by using the most common simplified spherical models - the fast rotator and the subsolar point approximations. We focus on the capability of the simple models to recover the active area fractions of the simulated complex objects.

\section{Description of the thermophysical model}

In order to carry out this study, a basic thermophysical model has been adopted in order to estimate the water production rates of simulated cometary nuclei, assuming the volatile component of the nucleus to be crystalline water ice. The governing equation for a sublimating surface element is the heat transfer equation,

$\rho C_{\mathrm{H}_{2} \mathrm{O}}(T) \frac{\partial T}{\partial t}=\frac{\partial}{\partial z}\left(\kappa_{\mathrm{H}_{2} \mathrm{O}}(T) \frac{\partial T}{\partial z}\right)$,

where $T$ is temperature, $t$ is time, $z$ is the depth coordinate, and $\rho, C_{\mathrm{H}_{2} \mathrm{O}}, \kappa_{\mathrm{H}_{2} \mathrm{O}}$ are the density, heat capacity, and thermal conductivity of the porous ice, respectively. In the simulations we use $\rho=500 \mathrm{~kg} \mathrm{~m}^{-3}, C_{\mathrm{H}_{2} \mathrm{O}}=90+7.49 T \mathrm{~J} \mathrm{~kg}^{-1} \mathrm{~K}^{-1}$, and $\kappa_{\mathrm{H}_{2} \mathrm{O}}=567 h / T \mathrm{~W} \mathrm{~m}^{-1} \mathrm{~K}^{-1}$ (Klinger 1981), where a Hertz factor $h=0.1$ has been used in order to simulate the effects of porosity. Equation (1) is integrated down to a depth much larger than the thermal skin depth corresponding to a heating cycle of

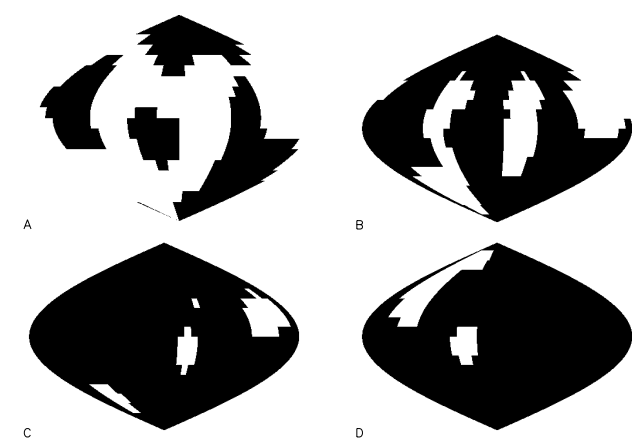

Fig. 2. Active area distributions used in this study. In these figures, white regions represent sublimating active areas and black regions represent the non-sublimating inactive areas. Distributions A, B, C and D have active areas constituting approximately $60 \%, 30 \%, 10 \%$, and $9 \%$ of the total surface area, respectively (see Table 1).

years. This allows us to assume a temperature gradient equal to zero as a lower boundary condition.

The upper boundary condition is given by the energy balance equation, where we assume that a fraction $(1-A)$ of the incident solar flux (unattenuated by the surrounding coma) is absorbed at the surface and is balanced by thermal radiation, sublimation of surface ice, and heat conduction into the nucleus,

$\frac{S_{\odot}(1-A)}{r_{\mathrm{h}}^{2}} J(\theta)=\epsilon \sigma T_{\mathrm{s}}^{4}+L Z\left(T_{\mathrm{S}}\right)-\left.\kappa_{\mathrm{H}_{2} \mathrm{O}}\left(T_{\mathrm{s}}\right) \frac{\partial T}{\partial z}\right|_{\mathrm{s}}$.

Here, $S_{\odot}$ is the solar constant, $A$ the albedo, $r_{\mathrm{h}}$ the heliocentric distance, $J(\theta)$ is a function of the solar zenith angle $\theta$ which describes the insolation, $\epsilon$ is the emissivity, $\sigma$ is the StefanBoltzmann constant, $L$ the latent heat (here considered as constant with a value of $2.68 \times 10^{6} \mathrm{~J} \mathrm{~kg}^{-1}$ ) and $T_{\mathrm{S}}$ stands for the temperature at the surface. The water production rate, $Z(T)$, can be approximated by the well-known Hertz-Knudsen formula multiplied by a factor $1-\alpha$ to correct for the recondensation of backscattered coma molecules (Crifo 1987). This author estimated the factor $\alpha$ as a function of the Mach number of outflowing gas, and found that $\alpha \approx 0.25$ is valid for a wide range of hydrodynamical situations. Taking this into account, the water sublimation rate can be estimated by

$Z(T)=(1-\alpha) Z_{\mathrm{HK}}(T)=0.75 \frac{p_{\mathrm{v}}(T)}{\sqrt{2 \pi m k T}}$,

where $m$ is the mass of a water molecule, $k$ is Boltzmann's constant, and $p_{\mathrm{v}}(T)$ is the saturated vapor pressure, which can be approximated by the semi-empirical expression given by Fanale \& Salvail (1984). 
Table 1. Active area fraction (percentage of the total area) for all combinations of nuclei shapes and activity patterns shown in Figs. 1 and 2. The active fraction has been calculated as $g_{\text {simulated }}=\sum_{i}^{n_{\mathrm{c}}} f_{i} s_{i} / \sum_{i}^{n_{\mathrm{c}}} s_{i}$.

\begin{tabular}{cccccc}
\hline \hline Object & Unif. & Distr. A & Distr. B & Distr. C & Distr. D \\
\hline 1 & 100 & 63 & 31 & 11 & 9 \\
2 & 100 & 62 & 29 & 8 & 8 \\
3 & 100 & 65 & 32 & 10 & 8 \\
Sphere & 100 & 60 & 28 & 8 & 9 \\
\hline
\end{tabular}

For computational reasons, the surface of a 3-D irregular nucleus is divided into small plane cells. Solving Eq. (1) for each active cell of the surface with $J=a_{i} \cos \theta$ in the energy balance equation (Eq. (2)), we obtain estimates for the surface temperature and the corresponding local water production rate $Z_{i}$. It must be noted that for a 3-D irregular object which is rotating, it is necessary to take shadow effects into account. This is done by giving the factor $a_{i}$ the value 1 when the cell is illuminated, and the value 0 when it is not (i.e., when in shadow and during nighttime. (See Gutiérrez et al. 2001 for details.) Thus, the total water production rate of a 3-D irregular body is approximately given by

$Q_{3 \mathrm{D}}=\sum_{i=1}^{n_{\mathrm{c}}} s_{i} f_{i} Z_{i}$,

where the summation is carried over all the cells the surface is divided into $\left(n_{\mathrm{c}}=1520\right)$ and $s_{i}$ is the area of cell $i$. In this model, active cells $\left(f_{i}=1\right)$ consist only of crystalline water ice, and inactive cells $\left(f_{i}=0\right)$ consist only of dust.

In the fast rotator approximation, the cosine of the solar zenith angle is averaged over the surface of the sphere, i.e., $J=\langle\cos \theta\rangle=1 / 4$ is used in Eq. (2). The solution to Eq. (1) in this case results in an isothermal nucleus, with a corresponding sublimation rate $Z_{\mathrm{fr}}$. Thus, the total water production of a partially active nucleus in the fast rotator approximation is

$Q_{\mathrm{fr}}=4 \pi r_{\mathrm{n}}^{2} Z_{\mathrm{fr}} g_{\mathrm{fr}}$,

where $g_{\mathrm{fr}}$ is the active area fraction of the nucleus, and $r_{\mathrm{n}}$ is its radius.

In the subsolar point approximation, the heat transport equation is solved with $J=1$ in Eq. (2), thus obtaining the subsolar point temperature and the corresponding gas production rate $\left(Z_{\mathrm{ss}}\right)$. By definition, the total gas production rate is then given by

$Q_{\mathrm{ss}}=\pi r_{\mathrm{n}}^{2} Z_{\mathrm{ss}} g_{\mathrm{ss}}$,

where $g_{\mathrm{ss}}$ is the active area fraction of the nucleus.

Several physical processes such as sub-surface sublimation and gas diffusion, surface erosion, dust mantle formation and layer absorption of solar energy have not been included in the model. Although this may influence the accuracy of our results, it is not expected that these simplifications invalidate our general conclusions if the same thermophysical model is adopted in the 3-D model ${ }^{1}$ and in the 1-D approximations. With this

\footnotetext{
1 For simplicity we use the nomenclature "3-D model" for a slowly rotating irregular nucleus, and "1-D approximations" for spherical
}

simple thermophysical description, the only difference between 1-D and 3-D models is the function $J(\theta)$ in the left hand side of the energy balance equation, Eq. (2).

\section{Nucleus shapes and simulations}

In order to build irregular surfaces which represent cometary nuclei, Gaussian random shapes (Peltoniemi et al. 1989; Muinonen 1996, 1996b, and 1998) have been used. This mathematical representation of irregular bodies has been applied by several investigators in the past to fit shapes of real minor bodies (Lagerros 1997; Muinonen \& Lagerros 1998). An important characteristic of these bodies is that they have a well defined mean radius, which in our calculations is set to the nominal value of $1 \mathrm{~km}$. In addition, different degrees of elongation and surface irregularity can be be considered, which makes the method ideal for this study. Figure 1 shows three Gaussian random shapes used in our study.

Regarding the activity pattern of the 3-D objects, we have considered two cases: uniform activity across the whole surface, and a mixture of active and inactive regions. In the latter case, several activity patterns have been generated by using randomized lognormal distributions in spherical coordinates. Only the surface facets characterized by a lognormal value greater than a predefined value are assumed to be active, while the rest of the surface is considered inactive.

Figure 2 shows four activity distributions that have been generated by using this method. Table 1 lists the corresponding total active area fractions for each of these activity patterns.

For each body of Fig. 1, both uniform activity and partial activity according to the patterns in Fig. 2 have been used to estimate the water production rate $Q_{3 \mathrm{D}}$ (Eq. (4)) for different spin axis orientations. $Q_{3 \mathrm{D}}$ have been compared to the total water production rates obtained with the two 1-D models (Eqs. (5) and (6)). The comparison is given in terms of the active area fraction that would be obtained by matching the 1-D model rates to the 3-D model rates, i.e., we have calculated

$g_{\mathrm{fr}}=\frac{\left\langle Q_{3 \mathrm{D}}\right\rangle}{4 \pi r_{\mathrm{n}}^{2} Z_{\mathrm{fr}}} \quad g_{\mathrm{ss}}=\frac{\left\langle Q_{3 \mathrm{D}}\right\rangle}{\pi r_{\mathrm{n}}^{2} Z_{\mathrm{ss}}}$

where $\left\langle Q_{3 \mathrm{D}}\right\rangle$ is obtained by averaging the production rate of the irregular bodies over one nucleus rotation.

These estimates of the active area fraction are then compared to the known value $g_{\text {simulated }}$ (see Table 1 ) of the active area fraction corresponding to each activity pattern of Fig. 2 .

In order to minimize the difference in absorbed energy between the irregular bodies and the corresponding 1-D model spheres, each sphere is given the same mean geometric albedo and mean geometric cross section as its irregular counterpart. The mean geometric cross sections of the irregular bodies were calculated by taking the average of the actual cross sections seen from 2592 different directions, uniformly distributed in spherical coordinates around each body. The corresponding mean radii are $1038.9 \mathrm{~m}, 1095.2 \mathrm{~m}$ and $1066.6 \mathrm{~m}$ for objects 1,2 , and 3 , respectively.

nuclei with illumination averaging. However, in order to avoid confusion we note that both models consider heat conduction in one spatial dimension only, which is parallel to the local surface normal. 

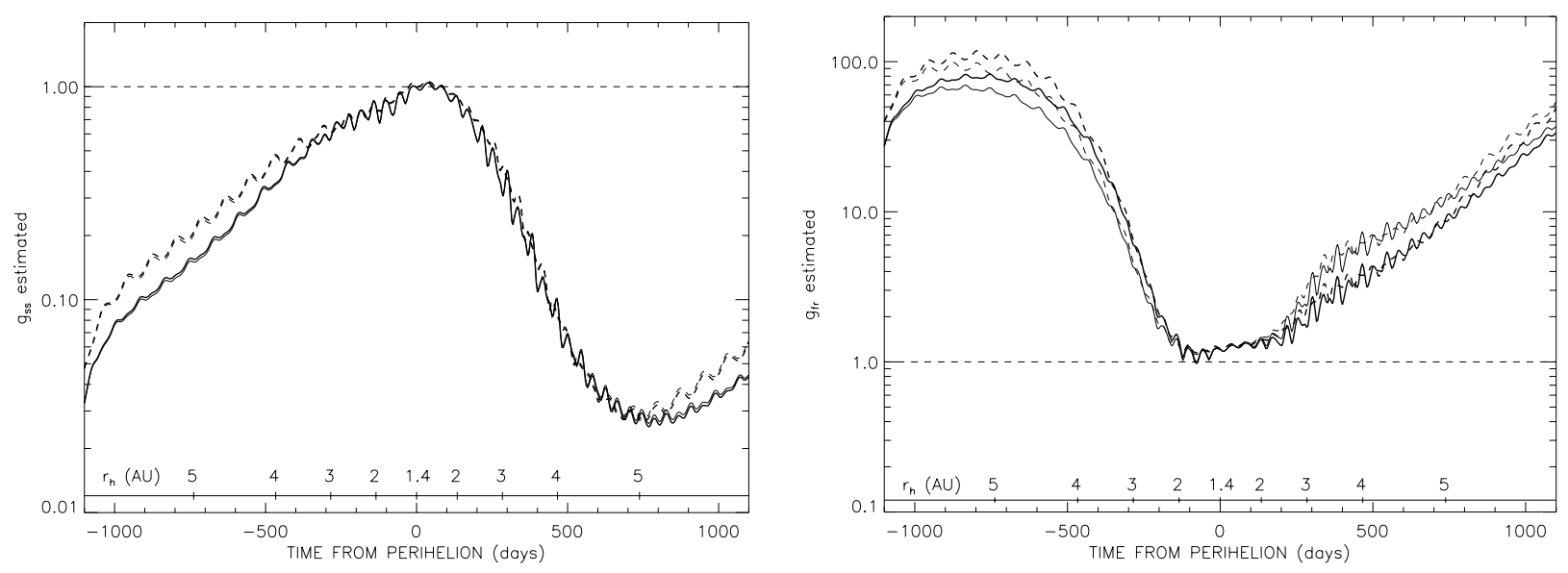

Fig. 3. Active area fractions estimated by comparing production rates of the subsolar point approximation (left) and the fast rotator approximation (right) to the rates of two uniformly active 3-D objects. Solid lines correspond to object 1 and dashed lines to object 2. Thin lines correspond to the case with a modest thermal conductivity and thick lines to the case with no thermal conductivity. The thin lines are practically indistinguishable from the thick lines in the plot to the left. In these simulations an orbit with $q=1.4$ and $e=0.6$ has been used. The spin axis orientation of the nucleus is $I=45^{\circ}$ and $\Phi=60^{\circ}$ and the spin period is $6 \mathrm{~h}$.

Most of the calculations have been done with $A=0.05$ and $\epsilon=0.95$, however, $A=0.1$ has also been considered. Concerning the orbital parameters, the orbit of Comet 46P/Wirtanen, and an orbit with $q=1.4 \mathrm{AU}$ and $e=0.6$ have been used in the calculations as typical orbits of short period comets.

With regard to the rotational parameters, a spin period of $6 \mathrm{~h}$ has been used. The simulations have been done for a complete set of spin axis orientations - we have considered 12 obliquity $(I)$ bins, running from $0^{\circ}$ to $180^{\circ}$ (the obliquity is the angle between the orbital plane and the equatorial plane of the object), and 12 argument $(\Phi)$ bins, running from $0^{\circ}$ to $360^{\circ}$ (the argument is the angle of the subsolar meridian at perihelion, measured from the ascending node), i.e., 144 spin axis orientations.

\section{Results and discussion}

\subsection{Simulations with thermal conductivity}

Several simulations have been done considering thermal diffusion into the interior of the nucleus, in order to study the influence of this term on the calculations. Some results of these simulations are shown in Fig. 3. The plot to the right in Fig. 3 shows the active area fraction obtained by applying the fast rotator approximation (Eq. (7), left) to the water production rates of two uniformly active irregular objects (objects 1 and 2) with and without thermal conductivity. The plot to the left shows the corresponding result for the subsolar point approximation (Eq. (7), right). Due to the uniform activity, it is expected that $g_{\mathrm{ss}}$ and $g_{\mathrm{fr}}$ both equals unity.

In Fig. 3, it can be seen that the fast rotator approximation underestimates the gas production rate and thereby overestimates the active area fraction, while the reverse is true for the subsolar point approximation. The errors grow fast with heliocentric distance.

It is clear that both 1-D models fail to recover the expected active area fraction at large heliocentric distances, even when the albedo and the mean radius of the nucleus are known exactly. At large heliocentric distances, the error can be of several orders of magnitude, and it depends strongly on the spin axis orientation and on the nucleus shape.

In the thermophysical model adopted here, the conductivity term generally plays a minor role, especially close to the Sun. Taking these first simulations into account, we have proceeded by only studying the water production rates at perihelion, and heat conduction into the nucleus has not been considered in the rest of the simulations.

\subsection{Simulations without thermal conductivity}

When we attempt to recover the active area fractions $g_{\text {simulated }}$ of various 3-D objects, by using the fast rotator and subsolar point approximations, we obtain errors ${ }^{2}$ (in percent) given by

$E_{\mathrm{fr}}=100\left(g_{\mathrm{fr}}-g_{\text {simulated }}\right) / g_{\text {simulated }}$

and

$E_{\mathrm{ss}}=100\left(g_{\mathrm{ss}}-g_{\text {simulated }}\right) / g_{\text {simulated }}$,

where $g_{\mathrm{fr}}$ and $g_{\mathrm{ss}}$ are given by Eqs. (7).

In Figs. 4 and 5, we show the values of $E_{\mathrm{fr}}$ and $E_{\mathrm{ss}}$ (valid at $r_{\mathrm{h}}=1.06 \mathrm{AU}$, i.e., the perihelion distance of $46 \mathrm{P} /$ Wirtanen) as functions of $\Phi$, for the nuclear shapes of Fig. 1 (including 3-D spherical bodies) and the A, C and D activity patterns of Fig. 2 . The uniformly active case is also shown. The vertical spread of data arises because different shapes and spin axis obliquities have been considered. In these plots, the complete gray region represents typical errors in the active area fraction estimate just due to the use of the simplified geometric treatment of the 1-D approximations.

2 The error when 1-D approximations are used to recover the active area from observational data, will depend also on the thermal model adopted. In this study, we are focused only in the error due to the geometric simplification used in the $1-\mathrm{D}$ approximations. 

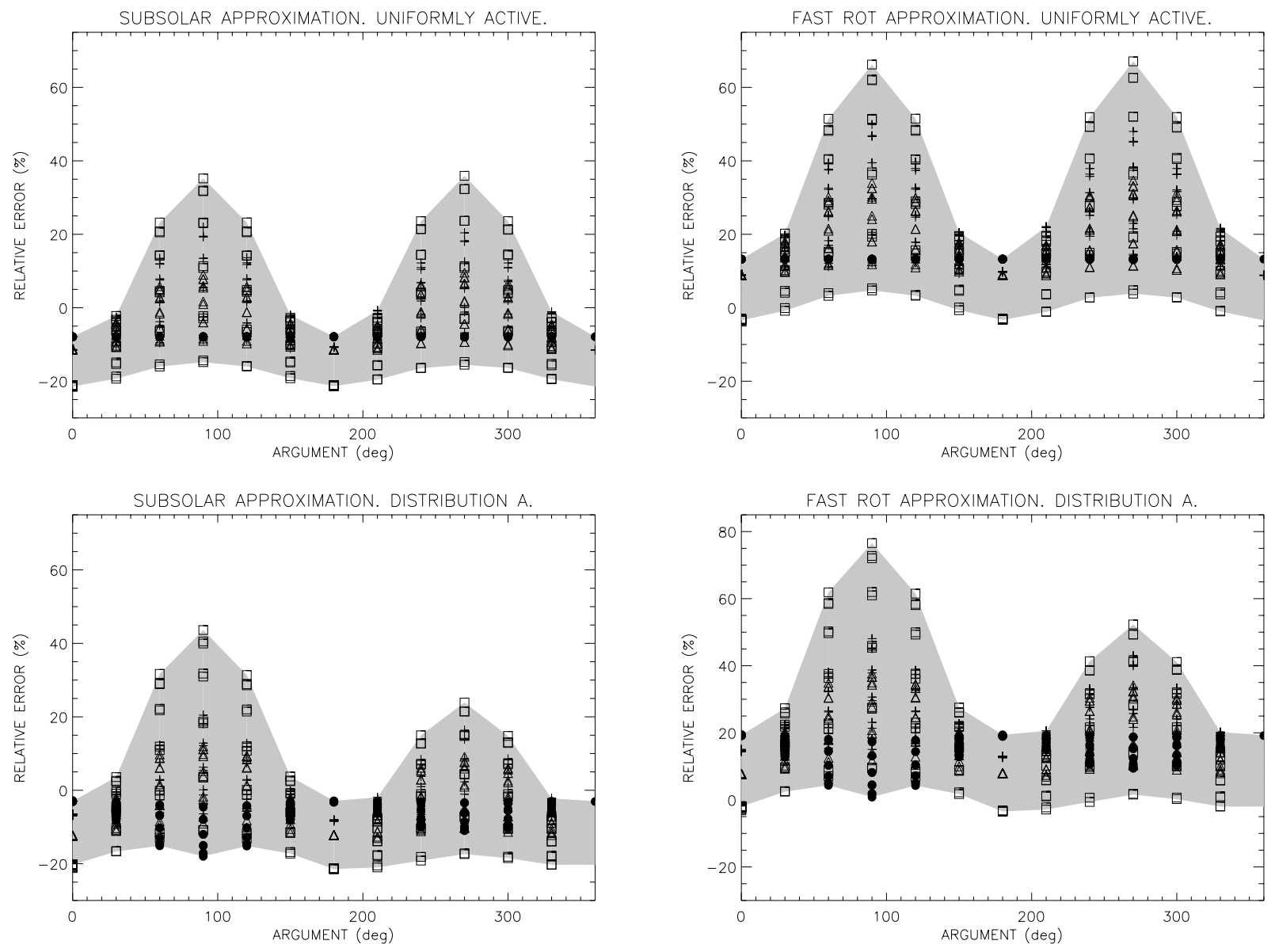

Fig. 4. Relative errors, as described in the text, in the estimate of the active area fraction of irregular nuclei using 1-D models, as function of the argument of the spin axis, $\Phi$. The left column shows the relative error obtained when the subsolar point approximation is used. The right column shows the error when the fast rotator approximation is used. The first row corresponds to uniformly active objects, and the second one to the activity pattern A. In all these plots, the symbols,$+ \Delta$, and $\square$ stand for objects 1,2 , and 3, respectively. Small bullets $(\bullet)$ show the relative error in the case when the body is indeed spherical. For each combination of shape, activity pattern and argument, we see a vertical spread of symbols, corresponding to the different obliquities. The complete gray region represents the typical error introduced by the 1-D approximations due to their simplified geometric treatment of the illumination. Note that the vertical scale is different in each plot.

The upper two diagrams in Fig. 4 contain the objects with uniform activity. In this case, $E_{\mathrm{ss}}<0$ and $E_{\mathrm{fr}}>0$ for the spherical object. In the first case, an artificial quenching is required since the subsolar approximation yields a too high sublimation rate $\left(g_{\mathrm{ss}}<g_{\text {simulated }}=1\right)$. In the second case, the fast rotator approximation produces less gas than the 3-D sphere, i.e., the latter object seems to be "hyper active" since the active area fraction is larger than unity. In this case, the relative errors for the spherical object do not change with $\Phi$ or $I$ as expected. However, the irregular objects have a strong $\Phi, I$ dependence, which can be understood by considering two different cases, $\Phi=0^{\circ}\left(\right.$ or $\left.180^{\circ}\right)$, and $\Phi=90^{\circ}$ (or $270^{\circ}$ ). In the first case, the subsolar point is always situated on the equator of the comet at perihelion, regardless of the obliquity $I$. Hence, only the nucleus shapes are responsible for the vertical spread in the data, for this argument. For the irregular objects, $E_{\mathrm{ss}}$ is even more negative than for the sphere, indicating that an irregular body with this orientation not only produces less gas than predicted by the subsolar approximation, but also less gas than a 3-D sphere. This is due to two reasons. (1) The mean cross section of the irregular body when viewed from the equatorial plane is smaller than the mean cross section of the whole body (it has been assumed that the bodies rotate around the axis of largest moment of inertia). Hence, less solar energy can be absorbed compared to the sphere and the gas production rate is reduced accordingly. (2) A larger number of the surface area elements are steeply oriented with respect to the Sun, compared to a sphere. As a consequence, the surface temperature is lower on average, and a larger fraction of the absorbed solar energy is lost as thermal reradiation, at the expense of water ice sublimation. This intrinsic reduction of the gas production rate also means that the irregular bodies are somewhat better represented by the fast rotator approximation than spheres $\left(E_{\mathrm{fr}}\right.$ tends to be reduced).

In the second case $\left(\Phi=90^{\circ}\right.$ or $\left.270^{\circ}\right)$, the spin axis nods straightly toward or away from the Sun, depending on $I$. In the extreme position $I=90^{\circ}$, the Sun is exactly above one of the poles of the comet. Since the polar cross section is larger than the mean cross section of the body, the gas production rate becomes substantially larger than predicted by both approximations (leading to large positive errors, $E_{\mathrm{ss}} \lesssim 35 \%$ and $\left.E_{\mathrm{fr}} \lesssim 65 \%\right)$. Compared to a sphere with the same cross section 

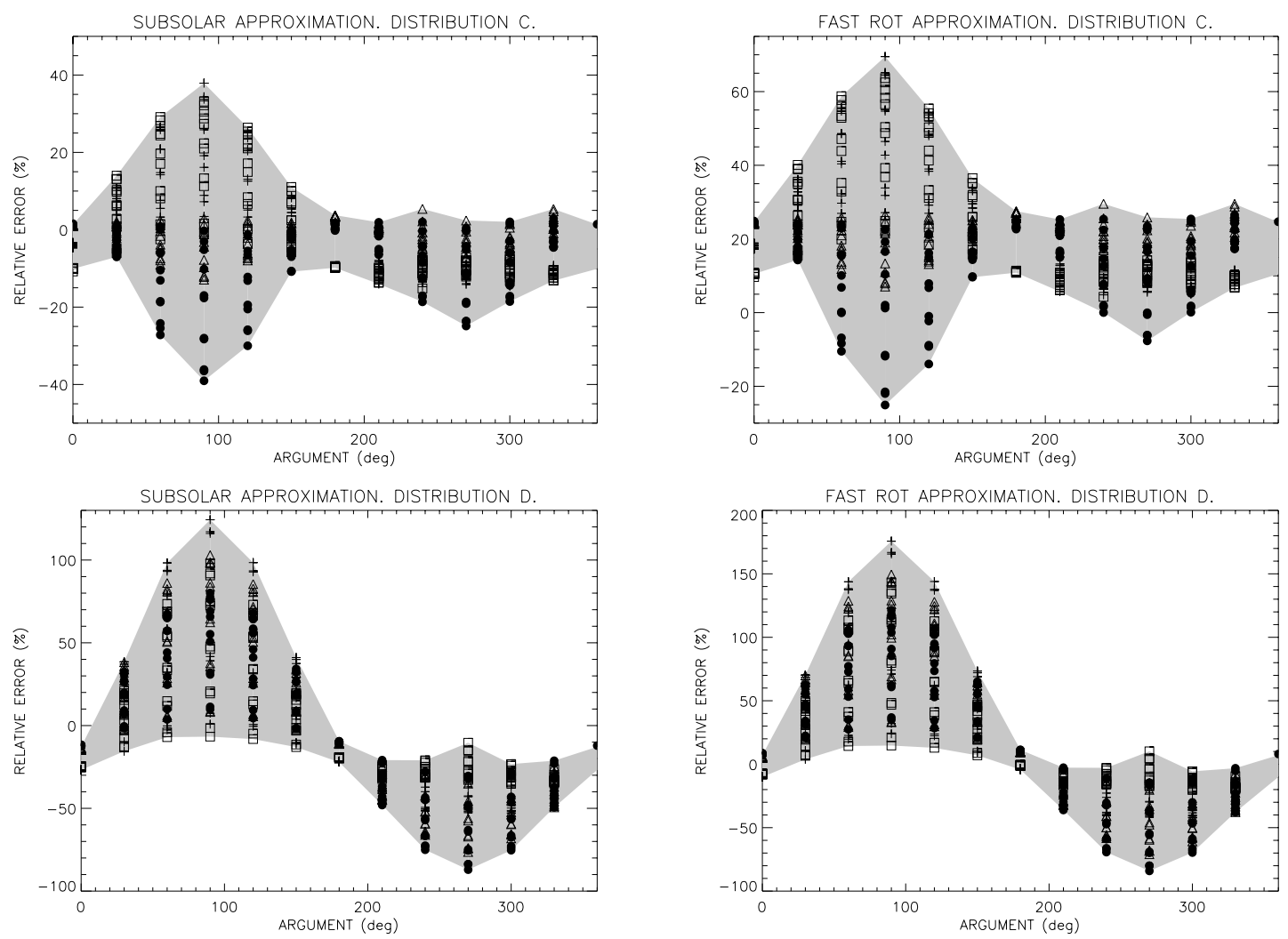

Fig. 5. This plot corresponds to Fig. 4, except that activity patterns $\mathrm{C}$ and $\mathrm{D}$ have been considered here.

as the pole-on irregular body, the latter still produces more gas, since the surface elements on average are illuminated at smaller incidence angles, than for the sphere.

When parts of a body are covered by inactive areas, the total sublimation rate depends strongly on the location of these areas on the body, and on the orientation of the spin axis. If the distributions $\mathrm{A}, \mathrm{B}$, and $\mathrm{C}$ are considered for the spherical object, it is found that $E_{\mathrm{ss}}$ generally is negative. As before, $g_{\mathrm{ss}}$ is then much smaller than $g_{\text {simulated }}$ since an extra compensation is required due to the intrinsically large production rate of the subsolar point approximation. As a result, the nucleus is believed to be more mantled than it really is. However, in some cases $E_{\mathrm{ss}} \gtrsim 0$, showing that the inactive areas are placed in such a way that the corresponding reduction of the total production rate is minimal. In this way, the inactive areas are "hiding" in poorly illuminated regions, and the subsolar approximation suddenly leads to a slight underestimation of the dust mantle coverage. If the fast rotator approximation is used, the situation is reversed. In most cases, the dust mantle coverage is underestimated, but if the inactive areas are placed where the effect on the global sublimation rate is strong, an overestimation is made (i.e., the nucleus is believed to have less surface ice than is the case).

The strong influence of the particular activity pattern is illustrated even more clearly by comparing the results for distribution $\mathrm{C}$ and $\mathrm{D}$ with each other (still focusing on spherical nuclei). In both cases, the active area fraction is $\sim 10 \%$, but Fig. 5 shows that $-40 \lesssim E_{\mathrm{ss}} \lesssim 5 \%$ for distribution C, while $-90 \lesssim E_{\mathrm{ss}} \lesssim 80 \%$ for distribution D. For the fast rotator approximation, the difference is even more dramatic, since
$-25 \lesssim E_{\mathrm{fr}} \lesssim 25 \%$ for distribution C, but $-90 \lesssim E_{\mathrm{fr}} \lesssim 125 \%$ for distribution D. Looking at Fig. 2, distribution $\mathrm{C}$ is characterized by a number of smaller active areas which are located on both the northern and southern hemisphere. Distribution D, on other hand, mainly consists of one large active region placed in the northern hemisphere.

Now focusing on the irregular objects with inactive regions, it is clear that the relative errors generally are more substantial than for uniformly active objects. The production rate of an object depends strongly on the specific shape, activity pattern and spin axis orientation. Errors can be very different depending on what hemisphere is illuminated. This is not due to a substantial difference in the size of the active regions between the hemispheres, but in their spatial distributions.

For distributions A and B ( $\left.g_{\text {simulated }} \gtrsim 30 \%\right),-20 \lesssim E_{\mathrm{ss}} \lesssim$ $55 \%$ for the irregular objects, while $-5 \lesssim E_{\mathrm{fr}} \lesssim 90 \%$. It is therefore fairly safe to say that an active area fraction obtained by using the fast rotator approximation, generally is an upper limit - the dust mantle coverage is underestimated. If a subsolar point model is used, the error probably becomes smaller, but it is more difficult to say if an over- or underestimation has been made.

For a very modest activity (distributions $\mathrm{C}$ and $\mathrm{D}$, with $\left.g_{\text {simulated }} \approx 10 \%\right)$, the errors can become very large $(-80 \lesssim$ $E_{\mathrm{ss}} \lesssim 120 \%$ and $-70 \lesssim E_{\mathrm{fr}} \lesssim 180 \%$ ), especially if most of the nucleus activity is concentrated to a single large area. In this case, there is no dramatic difference between the performance of the two approximations.

Several additional simulations have been carried out in order to study the effect of the albedo on the comparison. We 
have found that for an albedo value of 0.1 , the result of our comparison does not change.

Additional simulations have been done in order to study the effect of the perihelion distance on the estimate of the active area fraction. Using an orbit with $q=1.4$ and $e=0.6$, we found that, although the dispersions of $E_{\mathrm{fr}}$ and $E_{\mathrm{ss}}$ due to spin axis variations are similar regardless of the perihelion distance, the larger the perihelion distance, the lower the mean values of the errors. This means that the subsolar point approximation can underestimate the active area fraction even for large spin axis obliquities at large perihelion distances. For $q=1.4$, the fast rotator approximation always overestimates the active area fraction, as is the case for $q=1.06$.

\section{Summary and conclusions}

Water production rates obtained using the fast rotator and subsolar point approximations have been compared to the water production rates of several irregular bodies with different activity patterns on their surfaces. We have calculated the error which both approximations introduce, due to their oversimplified geometric treatment of shape and insolation, in the estimate of the active area fraction of a slowly rotating irregular body.

Test calculations showed that none of the 1-D approximations allow us to reliably estimate the active area of an irregular nucleus from the measured water production rate at a large heliocentric distance, although the mean albedo and mean geometric cross section of the nucleus are exactly known. At heliocentric distances larger than $2 \mathrm{AU}$, the relative difference between the active area estimate with a 1-D approximation and with a $3-D$ description is amply larger than $100 \%$.

At smaller heliocentric distances, exemplified by the perihelion distance of Comet 46P/Wirtanen $(q=1.06 \mathrm{AU})$, we found that the fast rotator approximation generally overestimates the active area fraction for irregular bodies. Only for very low activity $\left(g_{\text {simulated }} \lesssim 10 \%\right)$, substantial underestimates are likely. Typical errors due to an oversimplified geometric description of the order of $40-80 \%$ must be expected, even if the nuclear size, albedo and gas production rate can be measured rather accurately. The subsolar point approximation generally tends to overestimate the active area fraction. However, when the long axis of the body is perpendicular to the Sun-comet direction, rather substantial underestimates can also occur. The error $E_{\mathrm{ss}}$ is generally a bit smaller than that for the fast rotator approximation, $E_{\mathrm{fr}}$.

Even though the subsolar and fast rotator approximations can give a rough idea of the degree of activity, it may be dangerous to draw strong conclusions from such estimates. For example, Rickman \& Jorda (1998) used the subsolar approximation in order to estimate the active fraction of comet Wirtanen. They found that this comet, for plausible albedo values, could be hyper active. They pointed out several possible explanations for this effect, including additional sublimation from an inner coma of ice-rich grains, and a possible oversimplification of the thermophysical model. The present simulations show that $1-\mathrm{D}$ approximations are not accurate enough to prove such phenomena and that, for certain cases, the subsolar approximation can overestimate the active area fraction by a substantial amount for the orbit of comet Wirtanen. This observation cannot exclude hyper-activity per se, but makes it less likely.

We note that complex models have their value as realistic bench marks for simpler models in theoretical work, but we realize that they may be of small practical importance at present (e.g., for determining active area fractions of observed comets), since the nucleus shape and rotational characteristics only can be estimated for a few comets, at best. For practical purposes, we therefore recommend that the subsolar point approximation be used rather than the fast rotator approximation, but keeping in mind the inherent errors when the physical properties of real nuclei are discussed.

Acknowledgements. This research has been partially supported by the Spanish Ministerio de Ciencia y Tecnología under contract PNE001/2000-C-01. Davidsson acknowledges financial support from the graduate school of Advanced Instrumentation and Measurements (AIM), supported financially by the Foundation for Strategic Research (SSF).

\section{References}

Benkhoff, J., \& Huebner, W. F. 1995, Icarus, 114, 348

Benkhoff, J. 1999, Planet. Space Sci., 47, 735

Capria, M. T., Capaccioni, F., Coradini, A., et al. 1996, Planet. Space Sci., 44, 987

Crifo, J. F. 1987, A\&A, 187, 438

Davidsson, B. J. R., \& Skorov, Y. V. 2002, Icarus, 159, 239

de Almeida, A. A., Singh, P. D., \& Huebner, W. F. 1997, Planet. Space Sci., 45, 681

de Sanctis, M. C., Capaccioni, F., Capria, M. T., et al. 1999, Planet. Space Sci., 47, 855

Enzian, A., Cabot, H., \& Klinger, J. 1997, A\&A, 319, 995

Fanale, F. P., \& Salvail, J. R. 1984, Icarus, 60, 476

Fanale, F. P., \& Salvail, J. R. 1987, Icarus, 72, 535

Fink, U., Hicks, M. D., Fevig, R. A., \& Collins, J. 1998, A\&A, 335, L37

Gutiérrez, P. J., Ortiz, J. L, Rodrigo, R., \& López-Moreno, J. J. 2001, A\&A, 355, 326

Klinger, J. 1981, Icarus, 47, 320

Lagerros, J. S. V. 1997, A\&A, 325, 1226

Möhlmann, D. 1999, Planet. Space Sci., 47, 971

Muinonen, K. 1996, EM\&P, 72, 229

Muinonen, K. 1996, J. Quant. Spectrosc. Radiat. Transfer., 55, 603

Muinonen, K. 1998, A\&A, 332, 1087

Muinonen, K., \& Lagerros, J. S. V. 1998, A\&A, 333, 753

Orosei, R., Capaccioni, F., Capria, M. T., et al. 1995, A\&A, 301, 613

Peltoniemi, J. I., Lumme, K., Muinonen, K., et al. 1989, Appl. Opt., 28, 4088

Prialnik, D., Egozi, U., Bar-Nun, A., Podolak, M., \& Greenzweig, Y. 1993, Icarus, 106, 499

Rickman, H., Fernández, J. A., \& Gustafson, B. Å. S. 1990, A\&A, 237,524

Rickman, H., \& Jorda, L. 1998, Adv. Space Res., 21, 1491

Sanzovo, G. C., de Almeida, A. A., Misra, A., et al. 2001, MNRAS, 326,852

Tancredi, G., Rickman, H., \& Greenberg, J. M. 1994, A\&A, 286, 659 